\title{
Farmacopuntura com acepromazina para tranquilização de suínos
}

\section{Pharmacopuncture with acepromazine to tranquilize swine}

\author{
Ana Maria Quessada ${ }^{1 *}$; Karina de Oliveira Drumond ${ }^{2}$; Dário Batista Filho ${ }^{3}$; \\ Roseli Pizzigatti Klein ${ }^{4}$; João Macedo de Souza ${ }^{5}$; Flávia Melo Barreto ${ }^{6}$
}

\section{Resumo}

Foi realizado um estudo para tranqüilizar suínos com subdose de acepromazina no acuponto yin tang, localizado no ponto médio frontal de uma linha traçada entre os cantos laterais dos olhos. $\mathrm{O}$ estudo foi delineado em quatro etapas, realizadas em dias diferentes, utilizando-se cinco suínos. Na primeira etapa (E1) administrou-se acepromazina por via intramuscular (IM), na dose de $0,03 \mathrm{mg} /$ $\mathrm{kg}$. Na segunda etapa (E2) administrou-se acepromazina na dose de 0,003mg/kg, IM. Na terceira etapa (E3) foi administrada acepromazina no acuponto yin tang, na mesma dose empregada em E2. $\mathrm{Na}$ última etapa (E4) foi introduzida agulha de acupuntura no acuponto yin tang. Vinte minutos após E1, todos os animais apresentaram decúbito espontâneo, ptose palpebral e sonolência. Em E2, apenas um animal teve decúbito espontâneo, três apresentaram sonolência e ptose palpebral. Na E3, quatro animais tiveram decúbito espontâneo e todos apresentaram sonolência e ptose palpebral. Em E4 ocorreu decúbito espontâneo e sonolência em todos os animais; dois tiveram ptose palpebral. Concluiu-se que a administração de acepromazina em subdose no acuponto yin tang tranqüiliza suínos. A introdução da agulha no acuponto yin tang também tranquilizou os suínos, mas com dificuldades técnicas, pois a agulha deve permanecer no acuponto durante o tempo requerido para traquilização.

Palavras-chave: Acupuntura, porco, sedação, subdose, tranquilizante

\begin{abstract}
In order to tranquilize swine with acepromazine subdose in yin tang acupoint (located in the medium point of a line joined lateral cantus of the eyes in frontal region) was made this study. It was delineated in four steps in different days with five pigs. In first step (S1) acepromazine was administrated at $0.03 \mathrm{mg} /$ $\mathrm{kg}$ by intramuscular route. In second step (S2), the same drug was administrated at $0.003 \mathrm{mg} / \mathrm{kg}$ at same route. In the third step (S3), acepromazine was administrated in yin tang acupoint at same dose utilized in S2. In the last step (S4) acupuncture needle was introduced in yin tang acupoint. Twenty minutes after S1, all animals presented spontaneous decubitus, palpebral ptosis, and sleepiness. In S2, only one animal had spontaneous decubitus and three showed palpebral ptosis, and sleepiness. In S3 all animals had palpebral ptosis, and sleepiness, and four spontaneous decubitus. In S4 occurred spontaneous decubitus, and sleepiness in all animals, two had palpebral ptosis. In conclusion, acepromazine at subdose in yin tang acupoint tranquilizes pigs. The acupuncture needle introduction in yin tang acupoint tranquilizes pigs too, but with technical difficulties because the needle must remain in acupoint for the necessary time.
\end{abstract}

Key words: Acupuncture, pigs, sedation, subdose, tranquilizant

\footnotetext{
${ }^{1}$ Prof ${ }^{a}$. de clínica cirúrgica veterinária na graduação e discussão de casos clínicos na pós-graduação da Universidade Federal do Piauí, UFPI. E-mail: quessadavet@gmail.com

${ }^{2}$ Pós-graduanda em nível de doutorado no curso Ciência Animal da UFPI. E-mail: karinadrumond@gmail.com

${ }^{3}$ Médico veterinário da Agência de Defesa Agropecuária do Piauí. E-mail: veterinario80@yahoo.com.br

${ }^{4}$ Prof ${ }^{a}$ de clínica médica de cães e gatos no curso de Medicina Veterinária da UFPI. E-mail: roselipizzik@uol.com.br

${ }^{5}$ Prof. de diagnóstico por imagem no curso de Medicina Veterinária da UFPI. E-mail: smacedo@ufpi.br

${ }^{6}$ Médica Veterinária da Agência de Defesa Agropecuária do Piauí. E-mail: laviamelobarreto@yahoo.com.br

* Autor para correspondência
} 


\section{Introdução}

Devido às semelhanças fisiológicas com a espécie humana, os suínos são considerados, dentro da pesquisa biomédica, como o mais compatível modelo experimental animal (MALAVASY; NYMAN, 2004; THURMON; SMITH, 2007). Apesar disso, as técnicas anestésicas nesses animais pouco tem se desenvolvido nos últimos 10 anos. Este fenômeno talvez esteja intimamente ligado à criação do tipo industrial, em que o animal, ao menor problema apresentado, desde que apto para o consumo, é encaminhado sumariamente ao abate. No entanto, há uma demanda para utilização de técnicas anestésicas para serem aplicadas em pequenas criações ou em reprodutores, machos ou fêmeas, que se tornam alvos de maiores atenções por sua importância no aprimoramento do rebanho ou como modelo biológico em cirurgia experimental (MASSONE, 2008).

Com o aumento deste uso de suínos como modelo experimental, a busca por novas técnicas de tranqüilização que permitam uma manipulação adequada destes animais é muito importante, pois estes apresentam desvantagens em relação às outras espécies, devido, principalmente, ao estresse sofrido durante as manipulações e transporte. Desta forma, podem ocorrer óbitos em decorrência do estresse (MASSONE, 2008). Por isso, o estudo e o desenvolvimento de protocolos anestésicos e analgésicos para facilitar o manuseio desses animais devem ser prioritários (MALAVASY; NYMAN, 2004).

A acepromazina é o derivado fenotiazínico mais comumente utilizado como tranqüilizante na Medicina Veterinária (ANDRADE et al., 2008). É indicada para tranqüilização de suínos (SPINOSA; GÓRNIAK, 2006; THURMON; SMITH, 2007; ANDRADE et al., 2008; MASSONE, 2008), com a dose variando de acordo com o grau de sedação desejado (HALL; CLARKE; TRIM, 2001) sendo de $0,03 \mathrm{mg} / \mathrm{kg}$ a $2 \mathrm{mg} / \mathrm{kg}$ (HALL; CLARKE; TRIM, 2001; SPINOSA; GÓRNIAK, 2006), não devendo ultrapassar 15mg (THURMON; SMITH, 2007). Esse agente possui ação sedativa, promovendo decúbito espontâneo, ptose palpebral e sonolência (GROSS, 2003). Produz, também, redução da freqüência respiratória, freqüência cardíaca e temperatura retal (ANDRADE et al., 2008).

O princípio da farmacopuntura baseia-se na injeção de subdoses de medicamentos em pontos de acupuntura (JOAQUIM, 2003). Em caninos (CARDOSO, 2006) e, em grandes animais, esta técnica reduz os efeitos indesejáveis, a quantidade de resíduos em alimentos de origem animal e os custos com tratamentos (LUNA et al., 2006).

$\mathrm{O}$ acuponto yin tang se localiza no ponto médio de uma linha traçada entre os cantos laterais dos olhos e se liga com a inervação frontal (FLEMING, 2006). É usado para sedação em eqüinos, utilizandose um décimo da dose convencional de xilazina (JOAQUIM, 2003). Estudo realizado em cães sugere uma redução de $30 \%$ da dose convencional de tiopental quando administrada uma dose subclínica de acepromazina $(0,01 \mathrm{mg} / \mathrm{kg})$ no acuponto yin tang. Em eqüinos, uma subdose de acepromazina aplicada no acuponto yin tang tem efeito sedativo mais potente do que a dose convencional sem efeitos colaterais importantes (LUNA et al.,2006). Em cães, a subdose de acepromazina aplicada no acuponto yin tang, se mostrou segura, não havendo reações consideradas adversas nos animais estudados (CARDOSO, 2006).

Objetivou-se com esse estudo observar a ocorrência de tranquilização em suínos após administração de subdose de acepromazina no acuponto yin tang.

\section{Material e Métodos}

Foram utilizados cinco suínos híbridos comerciais (cruzamento de Landrace e Large White), machos, de aproximadamente três meses de idade e peso, em média, de $30 \mathrm{~kg}$. Os suínos eram de propriedade do Centro de Ciências Agrárias da 
Universidade Federal do Piauí, onde foi realizado o estudo. Os animais foram submetidos a jejum sólido de 12 horas e hídrico de seis horas. Após contenção, os animais foram utilizados no estudo que consistiu em quatro etapas (E1, E2, 3e e E4). $\mathrm{Na}$ primeira etapa (E1) administrou-se $0,03 \mathrm{mg} / \mathrm{kg}$ de acepromazina 1\% (Acepran 1\%, Univet, São Paulo), por via intramuscular (IM). Três dias depois, realizou-se a E2 utilizando-se um décimo (1/10) da dose preconizada para E1 $(0,003 \mathrm{mg} / \mathrm{kg}, \mathrm{IM})$. Na terceira etapa (E3), realizada seis dias após E1, o tranqüilizante foi aplicado no acuponto yin tang, na mesma dose empregada em E2, com a introdução de uma agulha $26 \mathrm{G}$ acoplada a uma seringa de 1 $\mathrm{ml}$ (Figura 1). Três dias depois de E3, realizou-se $\mathrm{E} 4$, onde foram posicionadas agulhas coreanas $\mathrm{n}^{\mathrm{o}}$. $0,30 \times 15 \mathrm{~mm}$ no ponto yin tang por um período de 20 minutos (Figura 2). Os animais foram monitorados atentamente para que as agulhas não saíssem de suas posições durante todo o período desta última etapa.

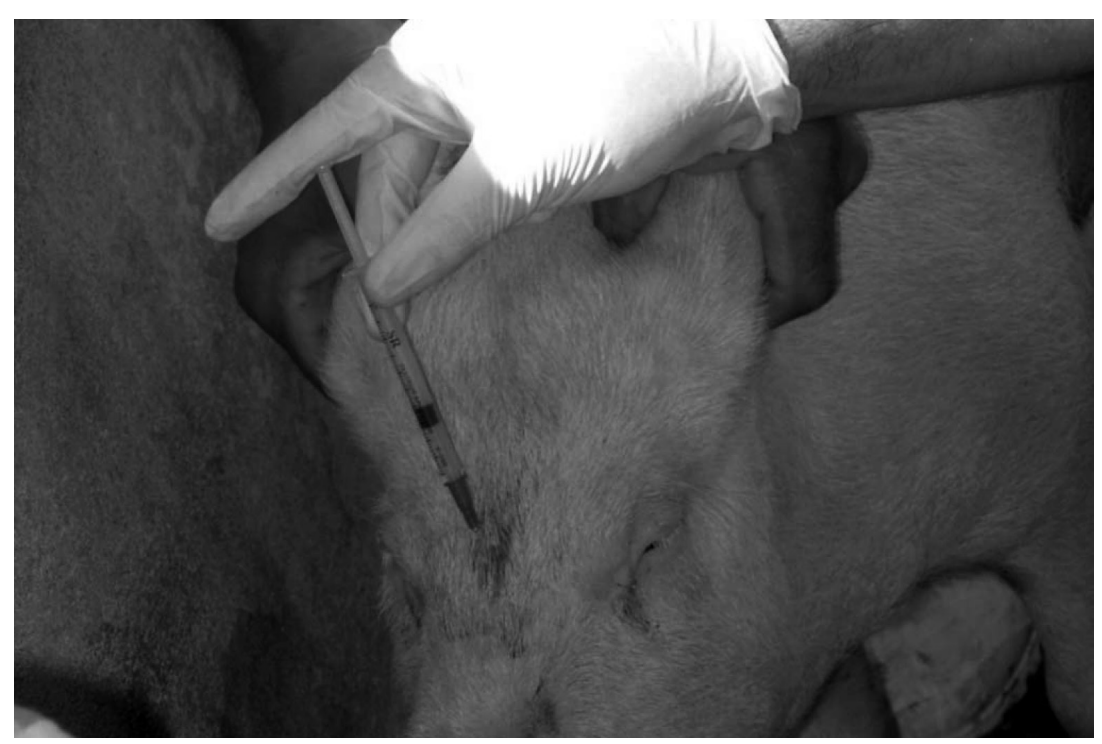

Figura 1. Administração de acepromazina em suíno, macho, Landrace x Large White, no acuponto yin tang.

Durante as quatro etapas foram aferidos os seguintes parâmetros fisiológicos: freqüência cardíaca (FC), freqüência respiratória $(f)$ e temperatura retal (TR). Todos os dados obtidos foram anotados em fichas individuais. Estas aferições foram realizadas em quatro momentos distintos: imediatamente antes dos tratamentos (M0), 20 (M1), 40 (M2) e 60 minutos (M3) após os procedimentos, respectivamente. Foi pesquisada a presença ou ausência de decúbito, sonolência, ptose palpebral e exposição peniana em todos os momentos analisados.

A FC foi obtida pela contagem dos batimentos cardíacos em um minuto, obtida por meio de estetoscópio. A $f$ foi obtida por meio da observação dos movimentos de expansão torácica em um minuto. A TR foi aferida utilizando-se um termômetro clínico veterinário introduzido diretamente no reto e mantido por um minuto para se proceder à leitura. Para observação do grau de sedação foi atribuído escore de acordo com a Tabela 1, adaptada de Cassu, Cordeiro e Rodenas (2005). 
Tabela 1. Escores, graus e sinais de sedação observados em animais domésticos.

\begin{tabular}{lcr}
\hline Escore & Grau de sedação & Sinais observados \\
\hline 0 & Ausência de & Sem alterações comportamentais; mantém-se atento aos fatores externos; tende \\
& sedação & a permanecer em estação; responde intensamente à manipulação. \\
1 & Sedação & Discreta alteração comportamental com sinais brandos de sedação; apresenta- \\
& moderada & se menos atento aos fatores ambientais externos; sonolento. \\
2 & Sedação & Sinais evidentes de sedação; pouca atenção aos fatores externos ambientais; \\
& satisfatória & sono; tendência à permanência em decúbito; mínima resposta à manipulação. \\
\hline
\end{tabular}

Fonte: Adaptado de Cassu, Cordeiro e Rodenas (2005).

A análise estatística da variável qualitativa (grau de sedação) foi realizada por análise de variância com aplicação de Teste de Friedman (AYRES et al., 2005). Para análise estatística das variáveis quantitativas (FC, $f$ e TR) foi realizada a análise de variância com aplicação do Teste $t$ de Student. O nível de significância utilizado em ambos os testes foi de $5 \%$.

O trabalho foi aprovado pelo comitê de ética em pesquisa animal da Universidade Federal do Piauí (Parecer 003/2008).

\section{Resultados e Discussão}

Foi observado que, 20 minutos após a aplicação de acepromazina em dose terapêutica (HALL; CLARKE; TRIM, 2001; SPINOSA; GÓRNIAK, 2006) por via intramuscular (E1), todos os animais apresentaram decúbito espontâneo, ptose palpebral e sonolência, os quais decorreram da ação sedativa da acepromazina (GROSS, 2003). Esses animais foram classificados com escore 2 (Tabela 2), pois tiveram sedação satisfatória.

Tabela 2. Escore médio do grau de sedação em suínos após cada protocolo de tranquilização. (Escore 0: ausência de sedação; Escore 1: sedação moderada; Escore 2: sedação satisfatória).

\begin{tabular}{lcccc}
\hline Animais & \multicolumn{4}{c}{ Etapas } \\
\cline { 2 - 5 } & E1 & E2 & E3 & E4 \\
\hline 1 & 2 & 1 & 2 & 1 \\
2 & 2 & 1 & 2 & 2 \\
3 & 2 & 0 & 1 & 1 \\
4 & 2 & 0 & 2 & 2 \\
5 & 2 & 1 & 2 & 2 \\
Total & 10,0 & 3,0 & 9,0 & 8,0 \\
Média & $2,0 \mathrm{a}$ & $0,6 \mathrm{~b}$ & $1,8 \mathrm{ab}$ & $1,6 \mathrm{ab}$ \\
\hline
\end{tabular}

E1: Acepromazina, $0,03 \mathrm{mg} / \mathrm{kg}$, IM (dose terapêutica)

E2: Acepromazina, $0,003 \mathrm{mg} / \mathrm{kg}$, IM (subdose)

E3: Acepromazina, $0,003 \mathrm{mg} / \mathrm{kg}$, acuponto yin tang (subdose)

E4: Aplicação de agulha de acupuntura no acuponto yin tang.

Médias seguidas por letras minúsculas diferentes, na linha, são estatisticamente distintas pela técnica da análise de variância, pelo teste de Friedman, a 5\% de significância. 
Quando se administrou a acepromazina em subdose por via intramuscular (E2), apenas um animal posicionou-se em decúbito de maneira espontânea e três apresentaram sonolência e ptose palpebral. Dois suínos foram classificados com escore 0 (ausência de sedação) e três com escore 1 (sedação moderada) (Tabela 2).

Após a administração da acepromazina em subdose no acuponto yin tang (E3), quatro animais postaram-se em decúbito espontâneo e todos apresentaram sonolência e ptose palpebral (Figura 3). Quatro animais foram classificados com escore 2 (sedação satisfatória) e um com escore 1 (sedação moderada) (Tabela 2).
Nos suínos submetidos apenas à acupuntura, estimulando-se o acuponto yin tang, ocorreu decúbito espontâneo e sonolência em todos os animais; dois tiveram ptose palpebral (Figuras 2 e $3)$. Neste grupo, três animais foram classificados com escore 2 (sedação satisfatória) e dois com escore 1 (sedação moderada) (Tabela 2).

Em equinos também se observa sedação quando se estimula o acuponto yin tang (LUNA et al., 2006). Embora tenha apresentado este efeito favorável, a técnica de se colocar apenas a agulha de acupuntura no acuponto yin tang foi considerada difícil de ser incorporada na prática rotineira de manipulação de suínos, porque a agulha deve permanecer no acuponto o tempo necessário para realizar o procedimento desejado no animal (Figura 2).

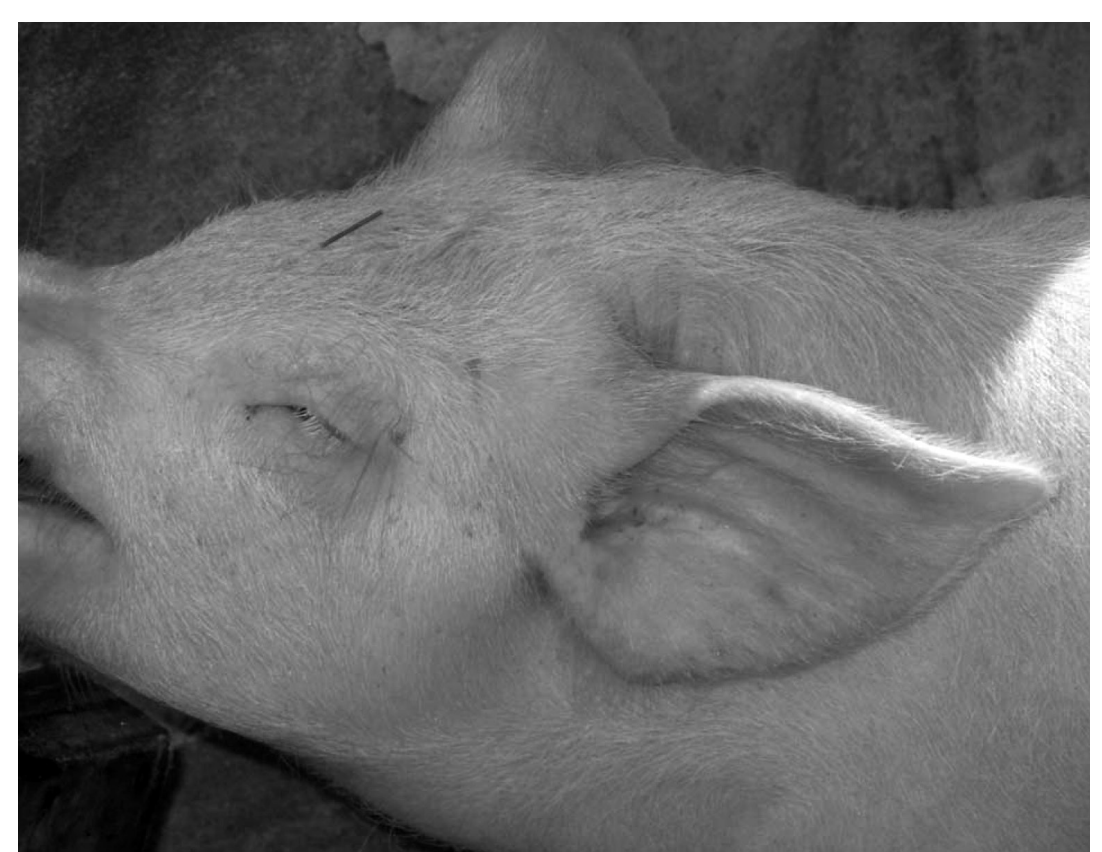

Figura 2. Suíno, macho, Landrace $\mathrm{x}$ Large White, tranquilizado com agulha de acupuntura no acuponto yin tang. Observar a ptose palpebral. 


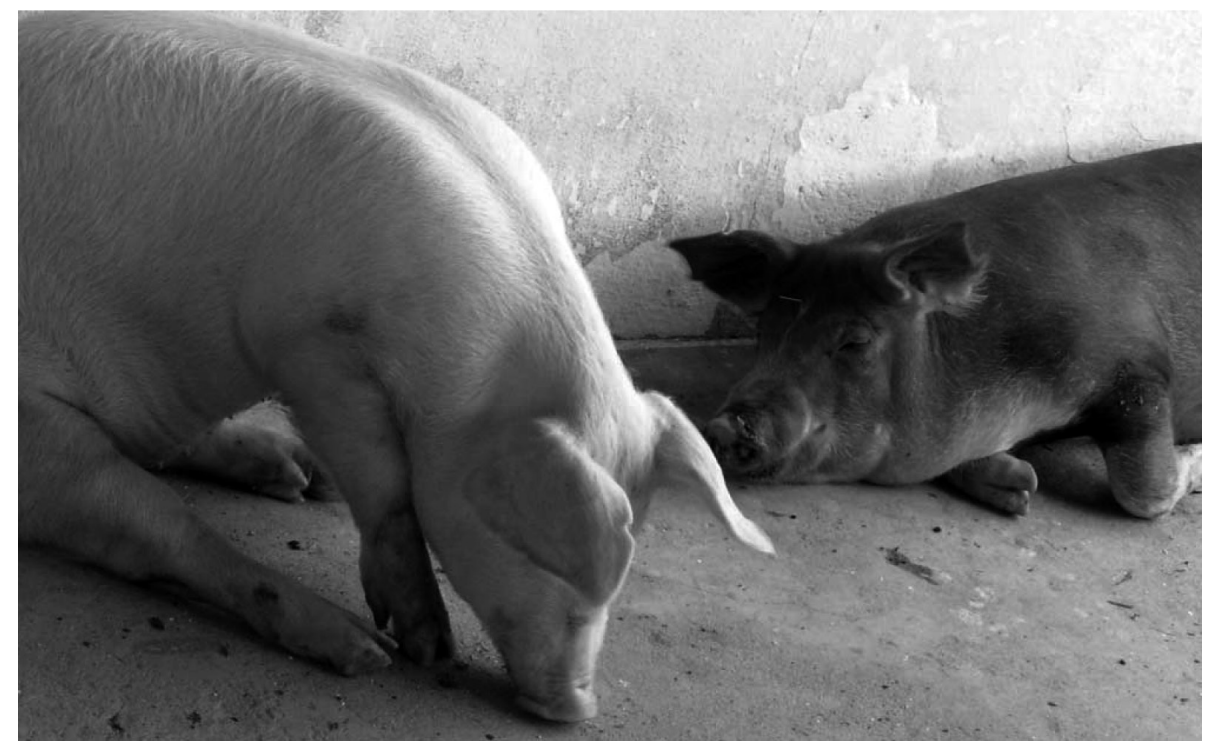

Figura 3. Suínos, machos, Landrace $x$ Large White, tranquilizados com subdose de acepromazina no acuponto yin tang (esquerda) e com aplicação de agulha de acupuntura no acuponto yin tang (direita). Observar sonolência, decúbito e ptose palpebral.

Houve diferença estatisticamente significativa entre E1 e E2 (Tabela 2), demonstrando que ocorreu sedação quando se administrou acepromazina em dose terapêutica por via intramuscular. Mas, quando se administrou o mesmo fármaco em subdose pela via intramuscular os animais não apresentaram sedação. Não ocorreu diferença estatisticamente significativa entre a sedação produzida pela acepromazina em dose terapêutica pela via intramuscular, a droga em subdose aplicada no acuponto yin tang e quando se aplicou apenas a agulha de acupuntura no acuponto yin tang. (Tabela 2).

$\mathrm{O}$ uso da acepromazina no acuponto yin tang revelou-se uma técnica viável de tranquilização em suínos. A execução foi fácil e rápida sem que os animais apresentassem sinais de grande desconforto. A acepromazina é indicada como tranquilizante em suínos (SPINOSA; GÓRNIAK, 2006; THURMON; SMITH, 2007; ANDRADE et al., 2008, MASSONE, 2008) com bons resultados (ANDRADE et al., 2008).

Embora a acepromazina possa reduzir as frequências cardíaca e respiratória e a temperatura retal (ANDRADE et al., 2008), estes parâmetros não apresentaram alterações estatisticamente significativas $(\mathrm{P}>5 \%)$, conservando-se nos valores considerados normais para a espécie (ANDERSSON; JÓNASSON, 2006; DETWEILER, 2006; REECE, 2006) durante todo o estudo, em todos os animais. Em caninos e eqüinos, experimentos com acepromazina administrada em subdose no acuponto yin tang também demonstraram não ocorrer alterações nos parâmetros clínicos referidos (CARDOSO, 2006; LUNA et al, 2006). A farmacopuntura, considerada eficiente em outras espécies (JOAQUIM, 2003; CARDOSO, 2006; LUNA et al., 2006) demonstrou ser bastante útil na tranquilização de suínos permitindo, ainda, redução de custos e de efeitos indesejáveis.

Salienta-se que a dose terapêutica aqui utilizada foi a menor referida na literatura para suínos (HALL; CLARKE; TRIM, 2001; SPINOSA; GÓRNIAK, 2006), tornando o procedimento ainda mais seguro.

Um aspecto inovador no estudo em questão é a espécie animal com a qual se trabalhou, pois não foram encontradas referências da utilização do acuponto yin tang em suínos. Os registros encontrados referem-se à espécie equina (JOAQUIM, 2003; FLEMING, 2006; LUNA et al., 2006) e canina (CARDOSO, 2006). 
Nas condições em que se realizou o estudo, observou-se que a administração de subdose de acepromazina no acuponto yin tang tranquiliza suínos com efeito semelhante à dose terapêutica por via intramuscular. A aplicação de agulha de acupuntura no acuponto yin tang também tranquiliza suínos, permitindo manipulação sem estresse. No entanto, este procedimento apresenta dificuldades técnicas, pois a agulha deve permanecer no acuponto durante o tempo de efeito requerido. Esta dificuldade técnica limita sua utilização na prática rotineira de manejo suíno.

\section{Referências}

ANDERSSON, B. E.; JÓNASSON, H. Regulação da temperatura e o ambiente térmico. In: SWENSON, M. J.; REECE, W. O. Dukes/fisiologia dos animais domésticos. 12. ed. Rio de Janeiro: Guanabara Koogan, 2006. cap. 55 , p. 805-813.

ANDRADE, S. F.; FANTONI, D. T.; CORTOPASSI, S. R. G.; ANDRADE NETO, J. P.; KANASHIRO. G. P. Terapêutica do sistema nervoso. In: ANDRADE, S. F. (Ed.). Manual de terapêutica veterinária. 3. ed. São Paulo: Roca, 2008. cap. 17, p. 433-518.

AYRES, M.; AYRES JUNIOR, M.; AYRES, D. L.; SANTOS, A. S. Bioestat. 4.0: aplicações estatísticas nas áreas das ciências biológicas e médicas. Belém: Ed. Sociedade Civil Mamirauá/MCT/Imprensa Oficial do Estado do Pará. 2005. 323 p.

CARDOSO, A. C. Tranquilização com acepromazina em cães através do acuponto yin tang. In: SEMINÁRIO DE INICIAÇÃO CIENTÍFICA DA UFPI, 15., 2006, Teresina. Anais...Teresina: UFPI, 2006. 1 CD-ROM.

CASSU, R. N.; CORDEIRO, M. O.; RODENAS, E. Avaliação do efeito sedativo e dose de indução anestésica do propofol sob diferentes medicações pré-anestésicas em gatos. Clín.Vet., São Paulo, v. 10, n. 58, p. 68-76, 2005.

DETWEILER, D. K. Regulação cardíaca. In: SWENSON, M. J.; REECE, W. O. (Ed.). Dukes/fisiologia dos animais domésticos. 12. ed. Rio de Janeiro: Guanabara Koogan, 2006. cap. 9, p. 157-169.
FLEMING, P. Atlas de transposição de acupuntura equina. In: SCHOEN, A. M. Acupuntura veterinária: da arte antiga à medicina moderna. 2. ed. São Paulo: Roca, 2006. cap. 31, p. 383-423.

GROSS, M. E. Tranquilizantes, agonistas $\alpha 2-$ adrenérgicos e agentes relacionados. In: ADAMS, $\mathrm{H}$. R. (Ed.). Farmacologia e terapêutica em veterinária. 8. ed. Rio de Janeiro: Guanabara Koogan, 2003. cap. 14, p. 249-284.

HALL, L. W.; CLARKE, K. H.; TRIM, C. M. Anaesthesia of the pig. In: (Ed.). Veterinary anaesthesia. 10. ed. London: Saunders, 2001. cap. 14, p. 367-383.

JOAQUIM, J. G. F. Curso de especialização em acupuntura veterinária. Botucatu: Universidade Estadual Paulista, 2003.12 p. (Apostila).

LUNA, S. P. L.; ANGELI, A. L.; FERREIRA, C. L.; LETTRY, V.; SCOGNAMILLOSZABÓ, M. Comparison of pharmacopuncture, aquapuncture and acepromazine for sedation of horses. Evid. Based. Complement. Alternat. Med., Oxford, v. 5, n. 1, p. 1-6, 2006.

MALAVASY, L. M.; NYMAN, G. Anestesia e analgesia em suínos, utilizados como modelo experimental. Rev. Cons. Fed. Med. Vet., Brasília, v. 10, n. 31, p. 23-28, 2004. Suplemento.

MASSONE, F. Técnicas anestésicas em suínos. In: . (Ed.). Anestesiologia veterinária, farmacologia e técnicas. 5. ed. Rio de Janeiro: Guanabara Koogan, 2008. cap. 13, p. 132-140.

REECE, W. O. Respiração nos mamíferos In: SWENSON, M. J.; REECE, W. O. (Ed.). Dukes/fisiologia dos animais domésticos. 12. ed. Rio de Janeiro: Guanabara Koogan, 2006. cap. 13, p. 103-134.

SPINOSA, H. S.; GÓRNIAK, S. L. Tranqüilizantes, relaxantes musculares de ação central e antidepressivos. In: SPINOSA, H. S.; GÓRNIAK, S. L.; BERNARDI, M. M. Farmacologia aplicada à medicina veterinária. 4. ed. Rio de Janeiro: Guanabara Koogan, 2006. cap. 14, p. $164-175$.

THURMON, J. C.; SMITH, G. W. Swine. In: TRANQULLI, W. J.; THURMON, J. C.; GRIMM, K. A. (Ed.). Lumb \& Jones'veterinary anesthesia and analgesia. 4. ed. Ames: Blackwell, 2007. cap. 29, p. 747764. 
